$$
\begin{aligned}
& \text { 震 }
\end{aligned}
$$

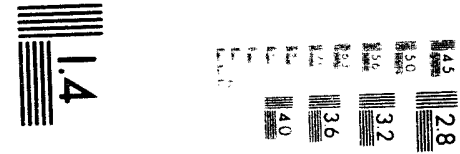

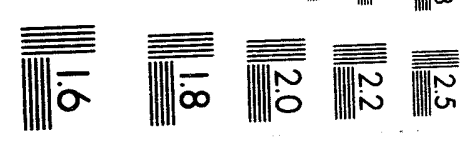



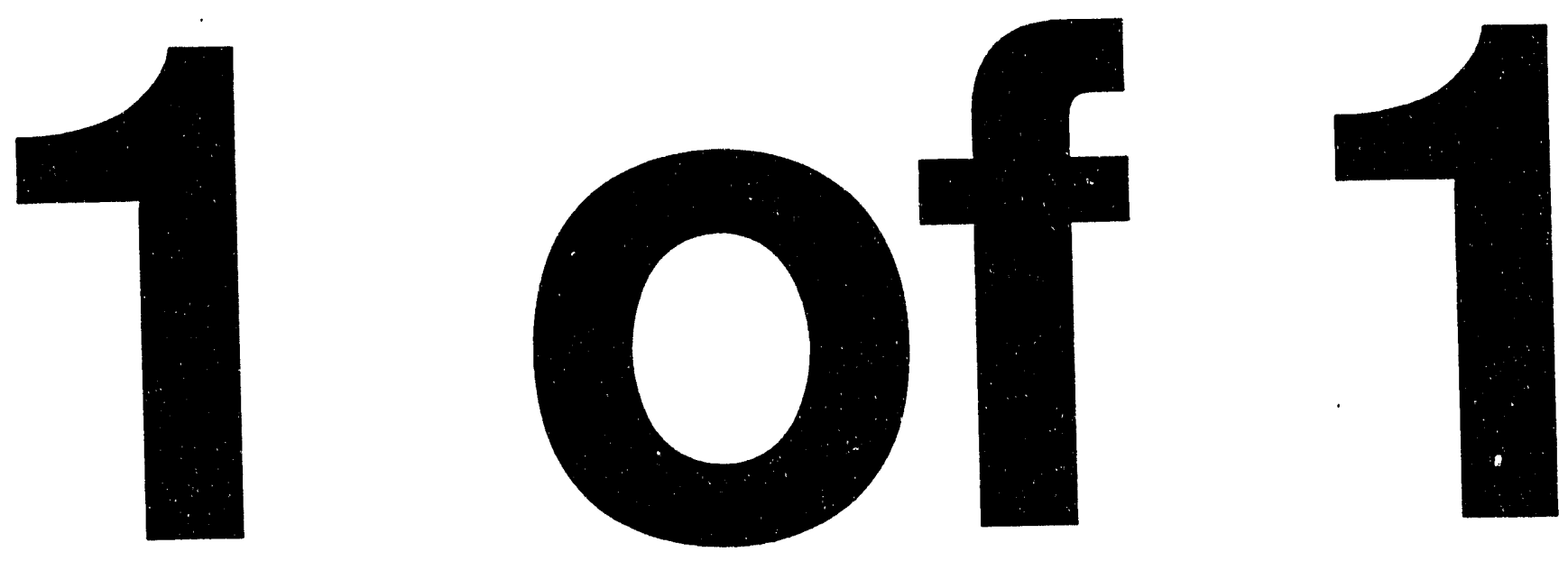


\section{UNIVERSITY OF WISCONSIN}

\section{Center for Plasma Theory and Computation REPORT}

Stability of Tearing Modes in

Tokamak Plasmas

C. C. Hegna and J. D. Callen

Nuclear Engineering \& Engineering Thysics and Physics Departments

University of Wisconsin-Madison Madison, Wisconsin 53706

February 1994

UW-CP'TC 94-1

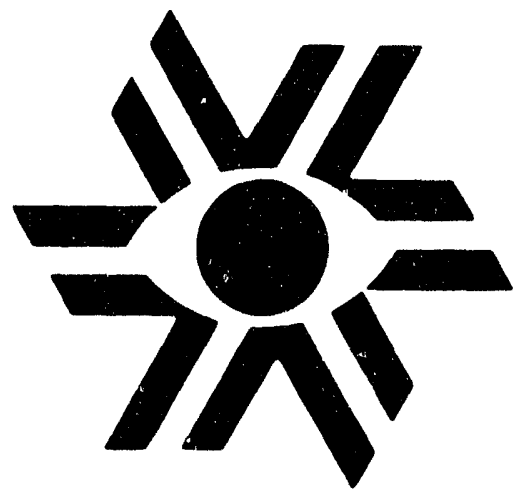

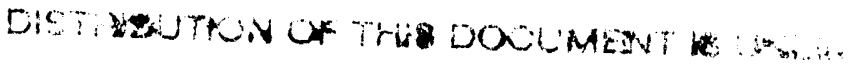

MAdisOn, Wisconsin 53706-1687 
This report has been reproduced directly from the best available copy.

Available to DOE and DOE contractors from the Office of Scientific and Technical Information, P.O. Box 62, Oak Ridge, TN 37831; prices available from (615) 576-8401, FTS 626-8401.

Available to the public from the National Technical Information Service, U.S. Department of Commerce, 5285 Port Royal Rd., Springfield, VA 22161.

This report was prepared as an account of work sponsored by an agency of the United States Government. Neither the United States Covernment nor any agency thereof, nor any of their employees, makes any warranty, express or implied, or assumes any legal liahility or responsibility for the accuracy, completeness, or usefulness of any information, apparatus, product, or process disclosed, or represents that its use would not infringe privately owned rights. Reference herein to any specific commercial product, process, or service by tradename, trademark, manufacturer, or otherwise, does not necessarily constitute or imply its endorsement, recommendation, or favoring by the United States Government or any agency thereof. The views and opinions of authors expressed herein do not necessarily state or reflect those of the United States Government or any agency thereof. 


\title{
Stability of Tearing Modes in
}

Tokamak Plasmas

\author{
C. C. Hegna and J. D. Callen \\ Departments of Nuclear Engineering and Engineering Physics and Physics \\ University of Wisconsin
}

Madison, WI 53706-1687

\begin{abstract}
The stability properties of $m \geq 2$ tearing instabilities in tokamak plasmas are analyzed. A boundary layer theory is used to find asymptotic solutions to the ideal external kink equation which are used to obtain a simple analytic expression for the tearing instability parameter $\Delta^{\prime}$. This calculation generalizes previous work on this topic by considering more general toroidal equilibria (however, toroidal coupling effects are ignored.) Constructions of $\Delta^{\prime}$ are obtained for plasmas with finite beta and for islands that have nonzero width. A simple heuristic estimate is given for the value of the saturated island width when the instability criterion is violated. A connection is made between the calculation of the asymptotic matching parameter in the finite beta and island width case to the nonlinear analog of the Glasser effect [A. H. Glasser, et al, Phys. Fluids 18, 875 (1975)].
\end{abstract}

PACS numbers: 52.35.Py, 52.30.Jb, 52.55.Fa, 52.55.Dy

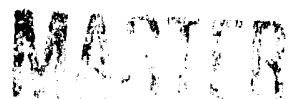




\section{Introduction}

Resistive magnetohydrodynamics (MHD) theory predicts the occurrence of current-driven tearing instabilities in tokamak plasmas. ${ }^{1}$ Resistive MHD models have been reasonably successful in characterizing a number of important macroscopic phenomena (Mirnov oscillations, locked modes, disruption physics, etc.) that have been observed in tokamaks for more than a decade. ${ }^{2}$ More recently, it has been observed that many of the high performance tokamak discharges exhibit low mode number MHD oscillations and the magnetic islands associated with these instabilities. ${ }^{3,4}$ The presence of these instabilities causes a degradation of the energy confinement time for these plasmas. The observed confinement degradation can be associated with the appearance of low mode number magnetic islands at rational surfaces. ${ }^{5}$ In particular, supershots on the Tokamak Fusion Test Reactor (TFTR) typically have $\mathrm{m} / \mathrm{n}=3 / 2$ or $\mathrm{m} / \mathrm{n}=4 / 3$ modes that occur, whereas the $m / n=2 / 1$ mode does not appear. ${ }^{3}$ Naively, this observation seems to be in contradiction to resistive MHD theory since one would expect the $m=2$ modes to be more unstable than the $m=3$ and $m=4$ modes. The ability to predict the appearance of these instabilities is an important issue for advanced tokamak operation scenarios.

In the linear resistive MHD theory of $\mathrm{m} \geq 2$ tearing modes, the plasma is assumed to be described by ideal MHD everywhere except a thin layer encompassing a magnetic surface where the safety factor is rational. The theory of asymptotics is then used to construct inner and outer region solutions which are then matched to link the nonideal inner layer solution to the outer solution. The outer region solution, where the effects of resistivity and inertia are small, is usually computed by numerically integrating the exterior kink equation. ${ }^{6}$ In resistive MHD, tearing mode stability is determined through the asymptotic matching parameter $\Delta^{\prime}$, which is constructed from the "exterior" ideal region 
solutions. For plasmas with no pressure gardient $(\nabla p=0)$, an energy integral can be constructed which demonstrates that the change in magnetic energy is proportional to $\Delta^{\prime}$ (the jump discontinuity in the logarithmic derivative of the perturbed magnetic potential). ${ }^{7}$ For a magnetic potential of the form $\Psi(\mathbf{x})=\psi(\mathrm{r})$ $\exp (\operatorname{im} \theta-\operatorname{in} \zeta)$, where $\theta$ and $\zeta$ are the poloidal and toroidal angles and it is assumed that the equilibrium magnetic surfaces are circles, the change in the magnetic energy is given by

$$
\mathrm{W}_{\mathrm{m}}=\frac{1}{2} \int \mathrm{d} \mathbf{x}\left\{\left|\nabla_{\perp} \Psi\right|^{2}+\frac{\mathrm{dj}_{||}}{\mathrm{dr}} \frac{1}{\mathrm{~B}_{\theta}} \frac{\mathrm{m}}{\mathrm{m}-\mathrm{nq}}|\Psi|^{2}\right\},
$$

where $j_{\mid l}$ is the parallel current, $B_{\theta}$ is the equilibrium poloidal magnetic field and $q(r)$ is the safety factor. Away from the rational surface the magnetic perturbation satisfies the Euler-Lagrange equation that results from minimizing the energy integral. However, the differential equation governing the magnetic potential has a singularity at the surface described by $q\left(r_{s}\right)=m / n$, which indicates that the general solution for $\psi$ has a discontinuity in the first derivative of $\psi$. Consequently, the change in magnetic energy is given by

$$
\begin{aligned}
W_{\mathrm{m}} & =\frac{1}{4} \lim _{\varepsilon \rightarrow 0}\left\{\mathrm{r}_{\mathrm{s}} \Psi * \frac{\mathrm{d} \Psi}{\mathrm{dr}}\left(\mathrm{r}_{\mathrm{s}}-\varepsilon\right)-\mathrm{r}_{\mathrm{s}} \Psi * \frac{\mathrm{d} \Psi}{\mathrm{dr}}\left(\mathrm{r}_{\mathrm{s}}+\varepsilon\right)\right\} \\
& =-\frac{1}{4} \mathrm{r}_{\mathrm{s}}\left|\Psi\left(\mathrm{r}_{\mathrm{s}}\right)\right|^{2} \Delta^{\prime},
\end{aligned}
$$

where we have chosen to consider only a single rational surface in a monotonically increasing $q$ profile. If $\Delta^{\prime}>0$, instability to tearing modes is indicated; then there exists a free-energy source for the resistive reconnection of magnetic field lines. ${ }^{6-8}$

It has also been shown that $\Delta^{\prime}$ remains a relevant stability quantity for $m \geq$ 2 tearing modes in collisionless and semi-collisional plasmas ${ }^{9}$ and also determines the ultimate nonlinear evolution of the magnetic island. ${ }^{10-13}$ 
For plasmas with finite beta $(\nabla p \neq 0)$ the solutions of the exterior region as they near the rational surface deviate from the zero beta case. The asymptotic matching parameter is obtained from the discontinuity of the ratio of the large to small solutions across the rational surface. ${ }^{14}$ Because of the finite beta modification of the exterior solutions near the rational surface, the analogous integration by parts of the magnetic energy integral [Eqs. (1) and (2) with pressure driven terms] produces a divergent integral. This divergence prevents using the primitive integral to construct a variational principle for determining non-ideal stability properties of finite beta plasmas. However, recently, Pletzer and Dewar have solved this problem by constructing a variational integral from an extended energy matrix. ${ }^{15}$ The extended matrix is constructed from contributions from both the potential energy integral of ideal MHD and from integrals over the large solutions. ${ }^{16}$ This method has significantly extended the usefulness of ideal MHD stability codes by creating an efficient method for using them to test for tearing mode stability.

In this paper, we use analytic methods to solve the exterior kink equation and construct the asymptotic matching parameter $\Delta^{\prime}$. The goal is to derive a simple, reliable analytic expression for $\Delta^{\prime}$ in terms of equilibrium plasma quantities that can be compared to experimental and numerical investigations of resistive MHD. A boundary layer theory is used to describe the ideal exterior region solution. The ideal boundary layer is centered on the resonant rational surface and is of radial extent $\mathrm{r}_{\mathrm{s}} / \mathrm{m}$ where we are treating $1 / \mathrm{m}$ as an asymptotically small number. In the next section we will first consider the case of a zero beta plasma and construct $\Delta^{\prime}$ at zero and finite magnetic island width. In Sec. III, nonzero beta effects are included and a similar construction of the matching parameter is made. We examine the case of a nonzero island width in 
a finite beta plasma in Section IV. A synthesis of these results and their implications is given in the final section.

\section{Tearing Stability of a $\beta=0$ Plasma}

The equilibrium configuration examined is assumed to be axisymmetric. As such, we write

$$
\mathbf{B}_{\mathrm{o}}=\mathrm{I}(\psi) \nabla \zeta+\nabla \zeta \times \nabla \psi,
$$

where $\zeta$ is the toroidal angle and $\psi$, the poloidal flux function, serves as a magnetic surface label. The poloidal angle magnetic coordinate $\chi$ is chosen such that

$$
\frac{\mathbf{B}_{0} \cdot \nabla \zeta}{\mathbf{B}_{0} \cdot \nabla \chi}=q(\psi),
$$

where $\mathrm{q}$ is the safety-factor and is assumed to be a monotonically increasing function of $\psi$. With this coordinate system the Jacobian has a simple form

$$
\mathrm{J}=(\nabla \psi \times \nabla \chi \cdot \nabla \zeta)^{-1}=\frac{\mathrm{qR}^{2}}{\mathrm{I}}
$$

where $\mathrm{R}$ is the major radius. The metric elements are defined by $\mathrm{g} \psi \psi=\nabla \psi \cdot \nabla \psi$, $\mathrm{g}^{\psi \chi}=\nabla \psi \cdot \nabla \chi, \mathrm{g}^{\chi \chi}=\nabla \chi \cdot \nabla \chi$. Since the equilibrium is axisymmetric, $\mathrm{g}^{\zeta \zeta}=\mathrm{R}^{-2}$, and $\mathrm{g}^{\psi \zeta}=\mathrm{g}^{\chi \zeta}=0$.

The ideal MHD equations are solved in the exterior region where inertia and nonideal effects are small. At $\beta=0$, the quasineutrality condition $\nabla \cdot J=0$ gives a relation for the linear perturbation

$$
\mathbf{B}_{\mathrm{o}} \cdot \nabla \frac{\tilde{j_{11}}}{\mathrm{~B}}+\tilde{\mathbf{B}} \cdot \nabla \frac{\tilde{j}_{110}}{\mathrm{~B}}=0
$$


where the perturbed magnetic field is written $\tilde{B}=\nabla \zeta \times \nabla \tilde{A}, \tilde{A}=A(\psi) \exp (\operatorname{im} \chi-$ $\operatorname{in} \zeta)$, and $m / n=q_{o}=q\left(\psi_{s}\right)$. The equilibrium parallel current is written $J \cdot B / B^{2}=$ $\sigma$. Equation (6) is now given by

$$
\frac{1}{\mathrm{~J}}\left(\mathrm{q} \frac{\partial}{\partial \zeta}+\frac{\partial}{\partial \chi}\right) \frac{\tilde{\mathrm{j}} \cdot \mathrm{B}}{\mathrm{B}^{2}}+\frac{1}{\mathrm{~J}}\left(\frac{\partial \sigma}{\partial \chi} \frac{\partial \mathrm{A}}{\partial \psi}-\frac{\partial \sigma}{\partial \psi} \frac{\partial \mathrm{A}}{\partial \chi}\right)=0,
$$

where Ampere's Law is used to construct the perturbed current and is given by

$$
\begin{aligned}
\mu_{\mathrm{O}}^{\frac{\tilde{\mathrm{I}} \cdot \mathrm{B}}{\mathrm{B}^{2}}} & =\frac{\mathrm{I}^{2}}{\mathrm{q}\left(\mathrm{I}^{2}+\mathrm{g}^{\psi \psi}\right)}\left[\frac{\partial}{\partial \psi}\left(\frac{\mathrm{J}}{\mathrm{R}^{2}} \mathrm{~g} \psi \psi \frac{\partial \tilde{\mathrm{A}}}{\partial \psi}+\frac{\mathrm{J}}{\mathrm{R}^{2}} \mathrm{~g} \psi \chi \frac{\partial \tilde{\mathrm{A}}}{\partial \chi}\right)\right. \\
& \left.+\frac{\partial}{\partial \chi}\left(\frac{\mathrm{J}}{\mathrm{R}^{2}} \mathrm{~g} \chi \chi \frac{\partial \tilde{\mathrm{A}}}{\partial \chi}+\frac{\mathrm{J}}{\mathrm{R}^{2}} \mathrm{~g}^{\psi \chi} \frac{\partial \tilde{\mathrm{A}}}{\partial \psi}\right)\right]-\frac{\mathrm{g} \psi}{\mathrm{I}^{2}+\mathrm{g}^{\psi \psi}} \frac{\partial}{\partial \zeta}\left(\frac{\mathrm{J}}{\mathrm{R}^{2}} \mathrm{~g} \chi \chi \frac{\partial \tilde{\mathrm{A}}}{\partial \chi}+\frac{\mathrm{J}}{\mathrm{R}^{2}} \mathrm{~g} \psi \chi \frac{\partial \tilde{\mathrm{A}}}{\partial \psi}\right) \\
& +\frac{\mathrm{g} \psi \chi}{\mathrm{I}^{2}+\mathrm{g} \psi \psi} \frac{\partial}{\partial \zeta}\left(\frac{\mathrm{J}}{\mathrm{R}^{2}} \mathrm{~g}^{\psi \psi} \frac{\partial \tilde{\mathrm{A}}}{\partial \psi}+\frac{\mathrm{J}}{\mathrm{R}^{2}} \mathrm{~g} \psi \chi \frac{\partial \tilde{\mathrm{A}}}{\partial \chi}\right) .
\end{aligned}
$$

The assumption that the toroidal magnetic field strength exceeds the poloidal field strength, $\left(\mathrm{g}^{\Psi \psi} \ll \mathrm{I}^{2}\right)$ allows the last two terms to be dropped and the perturbed current simplifies to

$$
\mu_{\mathrm{o}} \frac{\tilde{\mathbf{j}} \cdot \mathbf{B}}{B^{2}}=\frac{1}{q}\left[\frac{\partial}{\partial \psi}\left(\frac{q}{I} g \psi \psi \frac{\partial \tilde{A}}{\partial \psi}+\frac{q}{I} g \psi \chi \frac{\partial \tilde{A}}{\partial \chi}\right)+\frac{\partial}{\partial \chi}\left(\frac{q}{I} g \chi \chi \frac{\partial \tilde{A}}{\partial \chi}+\frac{q}{I} g \psi x \frac{\partial \tilde{A}}{\partial \psi}\right)\right],
$$

where Eq. (5) is used.

In general the metric elements in Eq. (9) are not flux functions, for example, $g^{\psi \psi}=g^{\psi \psi}(\psi, \chi)$. This complicates the calculation of tearing stability considerably since toroidicity couples all the rational surfaces with the same toroidal mode number. ${ }^{17}$ However, since we are seeking an equation for a single magnetic helicity, an averaging technique can be applied to Eqs. (7) and (9). We accomplish this by multiplying Eq. (7) by the phase factor $\exp (-\mathrm{im} \chi+\mathrm{n} \zeta)$ and then integrate over a field period by applying a $\oint_{\mathrm{dl}} / \mathrm{B}$ operator to Eq. (7). In these units $\oint \mathrm{d} l / \mathrm{B}=\oint \mathrm{Jd} \chi / 2 \pi$. The multiplication of the harmonic before the integration ensures that we obtain the resonant component. We write the 
perturbed magnetic field as a single helicity to avoid the problems of toroidal coupling, i. e. , $\tilde{\mathrm{A}}=\mathrm{A}(\psi) \exp (\mathrm{im} \chi-\mathrm{in} \zeta)$. These mode couplings tend to be small (order $\varepsilon^{2}$, where $\varepsilon$ is the inverse aspect ratio) in circular cross-section plasmas for large mode poloidal mode numbers; however, they may be very important for $\mathrm{m}$ $=1$, and $\mathrm{m}=2$ modes since the $\Delta^{\prime}$ for $\mathrm{m}=1$ goes as $\varepsilon^{-2}$. By applying this averaging technique, an ordinary differential equation for the mode amplitude is derived and given by

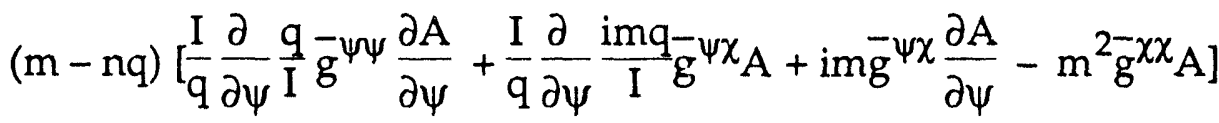

$$
\begin{aligned}
& -m I \mu_{0} \bar{\sigma}^{\prime} \mathrm{A}=0,
\end{aligned}
$$

where $\overline{\mathrm{g}}^{\psi \psi}=\oint(\mathrm{d} \chi / 2 \pi) \mathrm{g}^{\psi \psi}, \overline{\mathrm{g}}^{\chi \chi}=\oint(\mathrm{d} \chi / 2 \pi) \mathrm{g}^{\chi \chi}, \overline{\mathrm{g}}^{\psi \chi}=\oint(\mathrm{d} \chi / 2 \pi) \mathrm{g} \psi \chi$, and $\bar{\sigma}^{\prime}=$ $\oint(\mathrm{d} \chi / 2 \pi) \sigma^{\prime}$. The $\chi$-varying part of the equilibrium parallel current has been eliminated by the averaging. The $\chi$-dependent parts of the metric element $g^{\psi \chi}$ describe the Shafarnov shift and Pfirsch-Schlüter current effects, while the $\chi$ averaged portion of $\mathrm{g}^{\Psi X}$ is smaller. Motivated by this observation, we can further simplify the calculation by ignoring the $\overline{\mathrm{g}}^{\psi \chi}$ with respect to $\overline{\mathrm{g}}^{\psi \psi}$ and $\overline{\mathrm{g}} \chi \chi$. It is worth noting that these simplifications are not very good approximations in machines that have very small aspect ratios or highly shaped flux surfaces; however in such cases the toroidicity induced coupling of tearing mode eigenfunctions become important, ${ }^{17}$ effects that are outside the scope of the present study. Using this simplification, Eq. (10) reduces to

$$
(\mathrm{m}-\mathrm{nq})\left[\frac{\mathrm{I}}{\mathrm{q}} \frac{\partial}{\partial \psi} \frac{\mathrm{g}}{\mathrm{I}} \overline{\mathrm{g}} \psi \psi \frac{\partial \mathrm{A}}{\partial \psi}-\mathrm{m}^{2-} \mathrm{g}^{-\alpha \chi} \mathrm{A}\right]-\mathrm{mI} \mu_{\mathrm{o}} \bar{\sigma} \mathrm{A}=0 .
$$

Equation (11) is singular at the rational surface $m=n q$. Consequently, the general solution has a jump in the first derivative of $\mathrm{A}$ across the rational surface. Equation (11) is solved in two regions that are separated by the surface $\psi=\psi_{\mathrm{s}}$. 
The eigenfunction A satisfies boundary conditions at the magnetic axis and the conducting wall and is also demanded to be continuous across the rational surface.

In the present study we now treat $1 / \mathrm{m}$ as a small number and seek asymptotic solutions for A from Eq. (11). The solution A can be written in a perturbation series $A=\sum_{k}\left(A_{k} / m^{k}\right)$. Since $A$ varies more rapidly with $\psi$ than the equilibrium quantities, the two solutions for $\mathrm{A}$ to leading order in $1 / \mathrm{m}$ away from the rational surface are given by

$$
A=C_{1} \exp \left[\int d \psi m \sqrt{\frac{\bar{g} \chi \chi}{\frac{g}{g} \psi \psi}}\right]+C_{2} \exp \left[-\int d \psi m \sqrt{\frac{\bar{g} \chi \chi}{g^{\chi \alpha}}}\right],
$$

where $C_{1}$ and $C_{2}$ are constants multiplying the two solutions. As the magnetic axis is approached it is possible to show that $A \cong C_{1} r^{m}+C_{2} r^{-m}$ where $r$ is the "radial" distance from the magnetic axis. The condition that the solution is regular on the magnetic axis dictates that $C_{2}=0$ for the solution describing $A$ for $\psi<\psi_{s}$. In a similar manner, at large $\psi$ the solution is also given by the solutions $\mathrm{r}^{\mathrm{m}}$ and $\mathrm{r}^{-\mathrm{m}}$. Assuming the conducting wall is at infinity, $\mathrm{C}_{1}=0$ for the solution for $\psi>\psi_{s}$. To leading order in $1 / \mathrm{m}$, the solution to Eq. (11) is basically given by balancing the first two terms

$$
\begin{aligned}
& A^{+}=C_{+} \exp \left(-\int d \psi m \sqrt{\frac{g^{\chi \chi}}{g^{\psi \psi}}}\right) \text { for } \psi>\psi_{s}, \\
& A^{-}=C_{-} \exp \left(\int d \psi m \sqrt{\frac{g^{\alpha \chi}}{g^{\psi \psi}}}\right) \text { for } \psi<\psi_{s},
\end{aligned}
$$

where the superscripts $+(-)$ refer to $\psi-\psi_{s}>0(<0)$.

Clearly, this expansion procedure breaks down as we approach the rational surface, since for some value of $\psi$, the last term in Eq. (11) becomes comparable to the second term. Therefore, we now use a boundary layer theory 
on Eq. (11), where the "exterior" region of the ideal solution is given by the perturbation theory given above. The "interior" region of the ideal solution is then defined by the strained variable

$$
X=2 m\left(q-\frac{m}{n}\right) \sqrt{\frac{\bar{g} \chi \chi}{\bar{g}^{\psi \psi} q^{.2}}}
$$

where the terms inside the square root are evaluated at the rational surface. The strained variable $X$ is taken as an order unity quantity, so that the interior region describes the solution in a region of "radial" width $\mathrm{r}_{\mathrm{s}} / \mathrm{m}$ centered on the rational surface. We note parenthetically that the "natural" strained variable is written as a function of the deviation in q away from the rational surface, not the radial distance. We expand in this variable to avoid an unnatural singlarity that would arise in the second order expansion of the inner solution. The term involving the current gradient can be expanded as a function of $X$ near the rational surface. This expansion introduces a parameter at the rational surface given by

$$
\lambda=-\frac{\mathrm{Iq} \mu_{\mathrm{o}} \bar{\sigma}}{2 \mathrm{mq^{ \prime }}} \frac{1}{\sqrt{\bar{g}^{\chi \chi \chi_{\mathrm{g}} \psi \psi}}} \equiv-\left.\frac{\mathrm{rq}}{\mathrm{m}(\mathrm{dq} / \mathrm{dr})} \frac{\mu_{\mathrm{o}}}{\mathrm{B}_{\theta}} \frac{\mathrm{dj}_{\|}}{\mathrm{dr}}\right|_{\mathrm{r}=\mathrm{r}_{\mathrm{s}}},
$$

which is given by the value of the quantities at the rational surface and the last approximate form is the limit for circular flux surfaces in a cylinder. We will take this parameter to be order unity. For the case of a high- $\beta$ tokamak, an inverse aspect ratio expansion of the equilibrium can be made. ${ }^{18}$ For the case of a shifted circle equilbrium, finite $\varepsilon \beta_{\theta}$ corrections enter through the evaluation of the metric elements in Eq. (15a). The current gradient parameter for this case is given by

$$
\begin{aligned}
& \lambda \cong-\frac{r q}{m(d q / d r)} \frac{\mu_{0}}{B_{\theta}} \frac{d j_{\|}}{d r}{ }_{r}=r_{s} \times \\
& \left.\frac{1}{\sqrt{1+\left(v_{\beta} / 2 \rho\right)^{2}\left[1-4 \rho^{2}+5 \rho^{4}\right]}} \frac{1}{\sqrt{1+\left(v_{\beta} / 2 \rho\right)^{2}\left[1+9 \rho^{4}\right]}}\right|_{r=r_{s}}
\end{aligned}
$$


where $\rho=r / a, v_{\beta}=\beta_{t} q_{*}^{2} R_{0} / a, a$ is the minor radius, $R_{o}$ is the major radius, and $\beta_{t}$ and $q_{*}$ are the toroidal beta and kink safety factor as defined in Ref. 18. Since finite $v_{\beta}$ decreases $\lambda$, these finite beta/toroidal corrections have a small stabilizing effect,

The inner solution to leading order in $1 / \mathrm{m}$ is given by the solution of the differential equation

$$
\frac{\mathrm{d}^{2} \mathrm{~A}}{\mathrm{~d} \mathrm{X}^{2}}-\left[\frac{1}{4}+\frac{\lambda}{\mathrm{X}}\right] \mathrm{A}=0
$$

where the subscript indicating the order of the inner perturbation series is suppressed. The solutions to Eq. (16) are given by Whittaker functions. ${ }^{19} \mathrm{~A}$ number of properties of these special functions are given in Appendix A. The Whittaker functions $W_{-\lambda 0.5}(X)$ and $W_{\lambda / 0.5}(-X)$ are the two linearly independent solutions to Eq. (16). Asymptotic matching of the inner limit of the outer solution to the inner limit using Eqs. (13) and (A6) gives an expression for the eigenfunctions in the ideal interior region:

$$
\begin{aligned}
& A_{\text {inner }}^{+}=\Gamma(1+\lambda) W_{-\lambda / 0.5}(X) \text { for } X>0 \\
& A_{\text {inner }}{ }^{-}=\Gamma(1-\lambda) W_{\lambda / 0.5}(-X) \text { for } X<0,
\end{aligned}
$$

where continuity at $X=0$ has been demanded. Figure 1 shows yhe eigenfunctions for a range of values of the parameter $\lambda$. It shows that the difference in the derivative of $A$ across the rational surface increases with $\lambda$.

From the definition of $\Delta^{*}$ in zero beta plasmas, the asymptotic matching parameter is given by

$$
\begin{aligned}
\dot{\Delta}_{\mathrm{o}}^{\prime} & \equiv \lim _{\varepsilon \rightarrow 0}\left\{\frac{1}{\mathrm{~A}} \frac{\mathrm{dA}}{\mathrm{dr}}\left(\mathrm{r}_{\mathrm{s}}+\varepsilon\right)-\frac{1}{\mathrm{~A}} \frac{\mathrm{dA}}{\mathrm{dr}}\left(\mathrm{r}_{\mathrm{s}}-\varepsilon\right)\right\} \\
& =2 \mathrm{~m} \sqrt{\frac{-\chi \chi}{\mathrm{g} \chi}} \lim _{\varepsilon \rightarrow 0}\left\{\frac{1}{\mathrm{~A}} \frac{\mathrm{dA}}{\mathrm{dX}}(\varepsilon)-\frac{1}{\mathrm{~A}} \frac{\mathrm{dA}}{\mathrm{dX}}(-\varepsilon)\right\} .
\end{aligned}
$$


From Eqs. (17), (A7) and (A8), we obtain the result

$$
\Delta_{\mathrm{o}}^{\prime}=-2 \mathrm{~m} \sqrt{\overline{\mathrm{g}} \chi \chi} \lambda \pi \cot (\lambda \pi)
$$

where for circular flux surfaces $\sqrt{\frac{-\chi \chi}{\mathrm{g}}}=1 / \mathrm{r}_{\mathrm{s}}$. This result in the limit of circular flux surfaces has also been obtained by Strauss using a ballooning formalism. ${ }^{20}$ As $\lambda \rightarrow 0$, we recover the $\Delta_{o}^{\prime}=-2 \mathrm{~m} / \mathrm{r}_{\mathrm{s}}$ limit of large-m stable tearing modes. Equation (19) predicts the condition $|\lambda|>0.5$ or

$$
1 \frac{\operatorname{Iq} \mu_{o} \bar{\sigma}}{q^{\prime}} \mid \frac{1}{\sqrt{\bar{g}^{\chi x}-\bar{g} \psi \psi}} \mathrm{m}
$$

as the instability threshold for a tearing mode with poloidal mode number $\mathrm{m}$. For circular flux surfaces this inequality simplifies to

$$
\left|\frac{q r_{s} \mu_{0}}{q^{\prime} B_{\theta}} \frac{d j_{\|}}{d r}\right|>m \text {. }
$$

The threshold condition given in Eq. (21) has been predicted by previous authors for cylindrical plasmas. ${ }^{20-23}$ Equation (20) generalizes these predictions. In Appendix $B$, this stability condition is used to evaluate the stability criterion for the class of current profiles introduced in Ref. 6. Note that as $\lambda \rightarrow 1, \Delta^{\prime}$ approaches infinity. At $\lambda=1$, eigenfunction solutions can be constructed with the property $A(X=0)=0$. This suggests that $\lambda=1$ is the ideal stability threshold to lowest order in the $1 / \mathrm{m}$ expansion

Note that the instability condition in Eqs. (20) and (21) is given as a function of the local plasma quantities. It should be noted however, that this criterion is derived from a leading order expansion of the eigenfunction solution. The order $1 / \mathrm{m}$ corrections are given in Appendix $C$ where second derivatives of the current and $q$ profiles are taken into account. It has been noted for a particular current profile and q-profile, that Eq. (11) has an exact solution for cylindrical plasmas. ${ }^{24}$ 
Equation (20) predicts the linear theory threshold. However, if the resulting tearing instability produces a magnetic island that exceeds the linear tearing layer, the nonlinear theory of Rutherford ${ }^{13}$ should be used to describe the nonlinear evolution of the island. For this regime it is required that $\Delta^{\prime}$ be evaluated at finite island width. To compute this we use the finite island width definition of White et al. ${ }^{12}$ and Carreras et al. ${ }^{13}$ given by

$$
\Delta_{\mathrm{o}}^{\prime}(w) \equiv \frac{\frac{\mathrm{dA}}{\mathrm{dr}}\left(\mathrm{r}_{\mathrm{s}}+\mathrm{w}\right)-\frac{\mathrm{dA}}{\mathrm{dr}}\left(\mathrm{r}_{\mathrm{s}}-\mathrm{w}\right)}{\mathrm{A}\left(\mathrm{r}_{\mathrm{s}}\right)}
$$

to describe the asymptotic matching parameter, where $2 \mathrm{w}$ is the radial extent of the magnetic island. From the inner solution, Eq. (17), we obtain the relation

$$
\left.\Delta_{o}^{\prime}(w)=2 m \sqrt{\frac{-}{\mathrm{g} \chi}}\left\{\Gamma(1+\lambda) \mathrm{I}_{-\lambda}(\omega)+\Gamma(1-\lambda) \mathrm{I}_{\lambda}(\omega)\right]\right\},
$$

where

$$
\begin{aligned}
& \mathrm{I}_{\lambda}(\omega)=-\frac{1}{2} \mathrm{~W}_{\lambda / 0.5}(\omega)+\frac{\lambda}{\omega}\left[\mathrm{W}_{\lambda / 0.5}(\omega)-(1-\lambda) \mathrm{W}_{\lambda-1 / 0.5}(\omega)\right] \\
& \mathrm{I}_{-\lambda}(\omega)=-\frac{1}{2} \mathrm{~W}_{-\lambda / 0.5}(\omega)-\frac{\lambda}{\omega}\left[\mathrm{W}_{-\lambda / 0.5}(\omega)-(1+\lambda) \mathrm{W}_{-\lambda-1 / 0.5}(\omega)\right]
\end{aligned}
$$

are sums of Whittaker functions and $\omega \equiv 2 m \sqrt{\bar{g} \chi \chi} w$ is the normalized island width. As $w \rightarrow 0$, Eq. (19) is recovered from Eq. (23).

A saturated island width can be computed from the relation $\Delta_{0}^{\prime}\left(w_{\text {sat }}\right)=0$ if we ignore the extra quasilinear effect described by White, et al. ${ }^{12}$ The only free parameter in Eq. (23) is then the normalized current gradient parameter $\lambda$. Solving for the saturated island width yields a monotonically increasing function of $\lambda$, which can be given to reasonable accuracy by the simple relation for the island half-width when $\lambda$ exceeds the linear instability threshold

$$
\mathrm{w}_{\text {sat }} \cong \frac{4.09}{2 \mathrm{~m} \sqrt{-\overline{\mathrm{g}} \chi \chi}}\left(\lambda-\frac{1}{2}\right) .
$$


Figure 2 plots the solution of $\Delta_{\mathrm{o}}^{\prime}\left(\mathrm{w}_{\text {sat }}\right)=0$ as a function of $\lambda$ and the approximate solution, Eq. (25).

The definition used in Ref. 13 as outlined above for the saturated island width is not rigorously derived. However, it has been compared favorably to nonlinear numerical calculations of island saturation. ${ }^{12,13}$ A more proper use of singular perturbation theory can be used to obtain saturated island widths. ${ }^{25,26}$ However, as suggested in Ref. 25, the two approaches yield the same qualitative results and differ by only a small numerical difference.

\section{Tearing Stability of a $\beta \neq 0$ Plasma}

When $\nabla p \neq 0$ in the vicinity of a rational surface, an additional term is added to the exterior kink equation. From the quasineutrality condition, we obtain the relation $\mathbf{B} \cdot \nabla\left(\mathrm{J} \cdot \mathbf{B} / \mathrm{B}^{2}\right)=-\nabla \cdot \mathbf{J}_{\perp}$, where $\mathrm{J}_{\perp}$ depends on the pressure gradient through the diamagnetic current of the MHD equilibrium. This additional term describes the effect of the equilibrium pressure gradient and curvature. Including this term, the exterior kink equation is now given by

$$
\frac{I}{q} \frac{\partial}{\partial \psi} \frac{q}{I}-\bar{g} \psi \psi \frac{\partial A}{\partial \psi}-m^{2} \bar{g}^{\chi \chi} A-\frac{m}{m-n q} I \mu_{0} \bar{\sigma}^{\prime} A-\frac{m^{2}}{(m-n q)^{2}} I p^{\prime} \bar{J}^{\prime} A=0,
$$

where $p^{\prime}=d p / d \psi$ is the equilibrium pressure gradient and $\bar{J}^{\prime}=d \bar{J} / d \psi$ measures the surface averaged normal curvature. In cylindrical plasmas $\bar{J}^{\prime}>0$ which indicates a magnetic hill that is destabilizing to interchange instabilities, whereas a magnetic well is present in tokamak plasmas, $\left(\bar{J}^{\prime}<0\right)$. A more careful derivation of the pressure/curvature term yields the $\mathrm{E}+\mathrm{F}+\mathrm{H}$ terms of Glasser, Greene and Johnson. ${ }^{27}$

Although the last term in Eq. (26) is order $\beta$ and in principle small compared to the other terms of the equation, it has a large effect close to the rational surface. Near $\psi=\psi_{s^{\prime}}$ Eq. (26) has the approximate asymptotic form 


$$
\frac{d^{2} A}{d \psi^{2}}-\frac{\alpha}{\left(\psi-\psi_{s}\right)} A+\frac{1 / 4-D_{I}}{\left(\psi-\psi_{s}\right)^{2}} A=0
$$

where

$$
\begin{aligned}
& \alpha=-\frac{q_{0}}{q_{0}^{\prime}} \frac{I \mu_{0} \bar{\sigma}}{\bar{g} \psi \psi}, \\
& D_{I}=\frac{1}{4}+\frac{q_{0}^{2}}{q_{o}^{\prime 2}} \frac{I p^{\prime} J^{\prime}}{\bar{g} \psi \psi},
\end{aligned}
$$

are the current gradient and the Mercier term evaluated at the rational surface. The last term determines the asymptotic limit of the eigenfunction as it nears the rational surface. Specifically, the inner limit of the outer solution is given by

$$
A \cong C_{0}\left(\left|\psi-\psi_{s}\right|^{-v}+\Delta\left|\psi-\psi_{s}\right|^{1+v}\right) \text {, }
$$

where

$$
v=-\frac{1}{2}+\sqrt{D_{I}} \text {, }
$$

$\Delta$ is the ratio of the small to large solutions as the rational surface is approached, and $v-\beta$ for $\beta \ll 1$. It is presumed that the plasma is ideally stable $\left(D_{I}>0\right)$ so that $v$ is real. The discontinuity in the ratio of the small to large solutions is the asymptotic matching parameter,

$$
\left.\Delta_{\beta}^{\prime} \equiv\left[\Delta_{+}+\Delta_{-}\right] \overline{(g}^{\psi \psi}\right)^{1 / 2+v},
$$

where the plus (minus) sign refers to the solution for $\psi-\psi_{\mathrm{s}}>0(<0)$ and the metric factor is added so that $\Delta_{\beta}^{\prime}$ scales as the inverse length to the $1+2 v$ power. Clearly, this definition reduces to the zero beta $\Delta^{\prime}$ as $v \rightarrow 0$.

Following the same procedure as that used in the previous section, the asymptotic outer region of the ideal solution is given by the perturbation series whose first term is given by Eq. (13). Using the strained variable defined in Eq. 
(14), the inner region of the ideal solution is governed to leading order in $1 / \mathrm{m}$ by the equation

$$
\frac{\mathrm{d}^{2} \mathrm{~A}}{\mathrm{dX^{2 }}}-\left[\frac{1}{4}+\frac{\lambda}{\mathrm{X}}+\frac{\mathrm{v}+\mathrm{v}^{2}}{\mathrm{X}^{2}}\right] \mathrm{A}=0
$$

where $\lambda$ is given by Eq. (15). The two linearly independent solutions to Eq. (32) are given by the Whittaker functions $W_{-\lambda / 0.5+v}(X)$ and $W_{\lambda / 0.5+v}(-X)$ [see Appendix A and Ref. (19)]. From matching to the exterior solution we find the inner region solution

$$
\begin{aligned}
& A_{\text {inner }}^{+}=\frac{\Gamma(1+\lambda+v)}{\Gamma(1+2 v)} W_{-\lambda 0.5+v}(X) \text { for } X>0 \\
& A_{\text {inner }}^{-}=\frac{\Gamma(1-\lambda+v)}{\Gamma(1+2 v)} W_{\lambda / 0.5+v}(-X) \text { for } X<0 .
\end{aligned}
$$

As discussed in Appendix $A$, the Whittaker functions $W_{k, \mu}(X)$ with $\mu$ not a halfinteger can be written in a power series in $\mathrm{X}$ multiplying the leading order coefficients describing the small and large solutions. Note that the special value $\mu=1 / 2$ is the important case of zero beta that was described in the previous section and does not have a simple power series expansion. ${ }^{14}$ As $X \rightarrow 0$, the solutions of Eq. (34) have the asymptotic form of Eq. (30). Using Eq. (32), we obtain for the matching parameter

$$
\Delta_{\beta}^{\prime}=(2 m \sqrt{-\bar{g} \chi \chi})^{1}+2 v \frac{1}{2 v(1+2 v)} \frac{\Gamma(1-2 v)}{\Gamma(1+2 v)}\left\{\frac{\Gamma(1-\lambda+v)}{\Gamma(-\lambda-v)}+\frac{\Gamma(1+\lambda+v)}{\Gamma(\lambda-v)}\right\} .
$$

For $\beta \ll 1(v \ll 1), \Delta_{\beta}^{\prime}$ is given by

$$
\Delta_{\beta}^{\prime}=\Delta_{o}^{\prime}(2 m \sqrt{-\bar{g} \chi \chi})^{2 v}\left\{1+v[\Psi(\overline{1}+\lambda)-\Psi(1-\lambda)-2-4 \Psi(1)]+O\left(v^{2}\right)\right\},
$$

where $\Delta_{\mathrm{o}}^{\prime}$ is given in Eq. (19) and $\Psi(\mathrm{x})=\mathrm{d} \ln \Gamma(\mathrm{x}) / \mathrm{dx}$ is the Digamma-Psi function. 
A remarkable property of the stability condition given in Eq. (35) is that the threshold for instability $\left(\Delta_{\beta}^{\prime}=0\right.$ ) is given by $\lambda=1 / 2$ (as it is for $\beta=0$ ) and is independent of the value of $v$. Furthermore, for a given value of $v, \Delta_{\beta}$ increases monotonically with $\lambda$ just as in case of Sec. II. Figure 3 plots $\Delta_{\beta}^{\prime}$ as a function of $\lambda$ for a number of different values of $v$. As a practical matter since $v \equiv \beta \ll 1$, the exterior matching condition for linear stability theory is essentially unaffected by finite beta effects in MHD theory.

However, it is important to realize that the "Glasser" effect of resistive MHD theory manifests itself in the non-ideal boundary layer solution. ${ }^{27}$ The effect of pressure gradient/curvature in a resistive plasma changes the non-ideal boundary layer solution dramatically and consequently changes the linear instability threshold. We will examine this effect somewhat indirectly in the next section, when we address finite island width effects in non-zero pressure plasmas.

\section{Nonlinear stability of a $\beta \neq 0$ Plasma}

The linear stability of finite beta plasmas is determined by the ratios of the large and small solutions of the eigenmode equation. To determine the stability properties of a $\beta \neq 0$ plasma at small but nonzero island width, we need to define the necessary matching data. Motivated by the work of Pletzer and Dewar, ${ }^{14}$ we define the quantity

$$
\Delta_{\beta}^{\prime}(w) \equiv\left(\bar{g}^{\psi \psi \psi}\right)^{1 / 2+v} \frac{\left\{\left|\psi-\psi_{\mathrm{s}}\right|^{-v}\left[\frac{\mathrm{dA}}{\mathrm{d} \psi}-\frac{\mathrm{d} \hat{A}}{\mathrm{~d} \psi} \frac{\hat{\mathrm{A}}}{\mathrm{A}}\right]\right\}_{-w}^{+w}}{\lim _{\left|\psi-\psi_{\mathrm{s}}\right| \rightarrow 0}\left\{\left|\psi-\psi_{\mathrm{s}}\right|^{\nu} \mathrm{A}\right\}},
$$

which generalizes earlier definitions of the asymptotic matching parameter. The notation $\hat{A}$ denotes the large solution to the tearing mode equation. Since the large solution is responsible for the divergence of the energy integral, subtracting 
out this piece of the derivative eliminates the singularity at the rational surface. In the limit that the island width $w$ goes to zero it is easy to see that Eq. (37) reduces to the linear stability parameter of Eq. (32). For non-zero island widths, this quantity is not a rigorously defined quantity; however it does have the nice feature that it reduces to Eqs. (18), (22), and (32) in the appropriate limits. The Whittaker's function that solves the eigenmode equation is made up of a linear combination of the solutions $\mathrm{M}_{\mathrm{k} / \mu}$ and $\mathrm{M}_{\mathrm{k} /-\mu}$ [see Eqs. (A2) - (A5)]. The large solution needed for Eq. (37) is that part of $A$ given by $M_{k /-\mu}$ since it goes as $\mid \psi-$ $\left.\psi_{\mathrm{s}}\right|^{-v}$ as the rational surface is approached. Hence the large solution $\hat{A}$ is given by

$$
\begin{aligned}
& \hat{A}_{\text {inner }}^{+}=M_{-\lambda-0.5-v}(X) \text { for } X>0 \\
& \hat{A}_{\text {inner }}=M_{\lambda-0.5-v}(-X) \text { for } X<0 .
\end{aligned}
$$

From the eigenfunctions of Eq. (34) and (38), $\Delta_{\beta}^{\prime}(w)$ is computed and given by

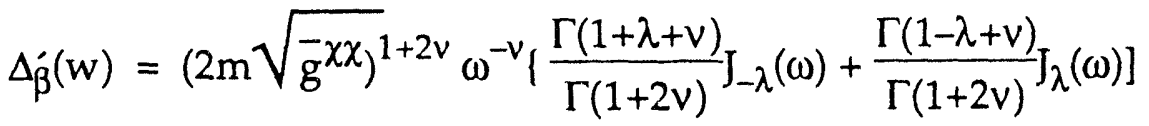

$$
\begin{aligned}
& \left.+\frac{\Gamma(1+2 v)}{\omega}\left[K_{-\lambda}(\omega)-K_{\lambda}(\omega)\right]\right\}
\end{aligned}
$$

where $\omega=2 \mathrm{~m} \sqrt{\frac{-}{\mathrm{g} \chi \chi}} \mathrm{w}, 2 \mathrm{w}$ is the radial extent of the island width and

$$
\begin{aligned}
J_{\lambda}(\omega)=- & \frac{1}{2} W_{\lambda / 0.5+v}(\omega) \\
& +\frac{1}{\omega}\left[\lambda W_{\lambda / 0.5+v}(\omega)-\left(\lambda-\lambda^{2}+v+v^{2}\right) W_{\lambda-1 / 0.5+v}(\omega),\right. \\
J_{-\lambda}(\omega)= & -\frac{1}{2} W_{-\lambda / 0.5+v}(\omega) \\
& -\frac{1}{\omega}\left[\lambda W_{-\lambda / 0.5+v}(\omega)-\left(\lambda+\lambda^{2}-v-v^{2}\right) W_{-\lambda-1 / 0.5+v}(\omega)\right],
\end{aligned}
$$




$$
\begin{aligned}
& K_{\lambda}(\omega)=M_{\lambda-0.5-v}(\omega) \frac{(\lambda-\omega / 2) M_{\lambda-0.5-v}(\omega)-(\lambda+v) M_{\lambda-1 /-0.5-v}(\omega)}{\Gamma(1-\lambda+v) W_{\lambda 0.5+v}(\omega)} \\
& K_{-\lambda}(\omega)=M_{-\lambda-0.5-v}(\omega) \frac{(\lambda+\omega / 2) M_{-\lambda-0.5-v}(\omega)-(\lambda-v) M_{-\lambda-1,-0.5-v}(\omega)}{\Gamma(1+\lambda+v) W_{-\lambda 0.5+v}(\omega)}
\end{aligned}
$$

All the previous results of this work are contained in Eq. (39) in the proper limits. As the island width becomes small, the last term in Eq. (39) is to leading order $-2 v / w$; consequently, the term $\Delta_{\beta}^{\prime}(w)$ can be simplified to be

$$
\Delta_{\beta}(w)=w^{-2 v}\left[\Delta_{o}^{\prime}(w)+\frac{2 v}{w}\right],
$$

where $\Delta_{o}^{\prime}(w)$ is the zero beta matching data, defined by Eq. (22). Note that for $\beta \neq$ 0 , the logarithmic derivative of A diverges as $w \rightarrow 0$, so that each term on the right hand side of Eq. (41) separately diverges at small island widths, but they cancel out in such a way as to give the ratio of large to small solutions, as defined by Eqs. (30)-(32).

Up to this point we have only discussed the ideal solution matching data. In order to obtain growth rates or an island evolution equation, the inner resistive layer matching data is needed (denoted $\Delta_{\text {res }}$ in this work). The matching is given by the equation $\Delta^{\prime}=\Delta_{\text {res }}$. As mentioned previously, the effects of pressure and curvature enter the resistive MHD problem through the interior region solution. In tokamak plasmas, these effects tend to be stabilizing and oppose destabilizing $\Delta^{-1} \mathrm{~s}^{27,28}$ In the nonlinear Rutherford regime ${ }^{10}$ the pressure gradient/curvature effect becomes important near the rational surface. ${ }^{29}$ In the work of Ref. 29 , the interior matching data for the Rutherford regime was computed for $\beta \neq 0$ plasmas. After matching to the exterior solution, an island evolution equation was derived and given by

$$
\Delta_{\dot{\beta}}^{\prime}=\Delta_{\text {res }}=w^{-2 v}\left(k_{1} \frac{\mu_{0}}{\eta} \frac{d w}{d t}-k_{2} \frac{D_{R}}{w}\right)
$$


where $\eta / \mu_{0}$ is the resistive diffusion rate, $k_{1}$ and $k_{2}$ are positive constants of order unity and $D_{R}$ is the resistive interchange parameter as defined in Ref. 26. Note that the pressure driven term $\left(D_{R}\right)$ appears in the interior matching data. Since finite plasma pressure is allowed in the interior region, the appropriate matching is to the difference of the small to large solutions in the ideal exterior region (denoted $\Delta_{\beta}^{\prime}$ in this work). However, suppose the pressure gradient was flattened in the interior resistive layer. This eliminates the pressure gradient term in the inner region solution and also changes the asymptotic properties of the inner solution. The resistive layer solution now matches to the logarithmic derivative of the ideal exterior solution, $\Delta_{\text {res }}=\Delta_{o}^{\prime}$. If we allow for pressure in the exterior solution, the logarithmic derivative diverges as described above. From Eq. (41), we can derive the island evolution equation for the magnetic island,

$$
\Delta_{\mathrm{res}}=\Delta_{\mathrm{o}}^{\prime}=\mathrm{w}^{2 v} \Delta_{\beta}^{\prime}-\frac{2 v}{\mathrm{w}} .
$$

In the Rutherford limit ${ }^{10}\left[\Delta_{\text {res }}=\left(k_{1} \mu_{o} / \eta\right)(d w / d t)\right]$, Eq. (43) yields the same equation as derived in Ref. 29 [see Eq. (42) above],

$$
k_{1} \frac{\mu_{o}}{\eta} \frac{d w}{d t}=\Delta_{\beta}^{\prime} w^{2 v}-\frac{2 v}{w} \text {. }
$$

For low $\beta, D_{R}$ and (-v) differ by only a small factor.

The difference between the matching data for finite beta and zero beta plasmas is naturally given by terms that goes as $2 v / w$, as indicated above. This has also been pointed out by Bishop et al., ${ }^{30}$ who solved for the linear eigenfunctions in an equilibrium that had a pressure profile that was arbitrarily flattened across a region that encompassed the rational surface. In their work, the matching data differed by a term that went inversely with the radial extent of 
the flatspot. For tearing modes that produce a finite island size, the width of this "flatspot" is proportional to the island width.

\section{Discussion}

An analytic theory for tearing mode stability with poloidal number $\mathrm{m} \geq 2$ in tokamaks has been introduced. A boundary layer theory was used on the ideal region of the plasma cross-section where the boundary layer is of width $\mathrm{r}_{\mathrm{s}} / \mathrm{m}$ centered on the mode rational surface. The resulting asymptotic eigenfunction solutions were used to construct the tearing mode matching parameter, $\Delta^{\prime}$, given in Eq. (19), which determines the stability properties of MHD tearing modes. This procedure yields a simple stability criterion given by Eq. (20), which generalizes earlier work on this subject to include toroidal equilibrium effects. The linear eigenmode is constructed for both pressure free and finite pressure equilibria. It is found that an equilibrium pressure gradient at the rational surface does not alter the parallel current gradient stability threshold, Eq. (20). Further, in realistic tokamak equilibria it does not dramatically change the numerical value of $\Delta^{\prime}$ [see Eqs. (35) and (36)]. As discussed in Appendix B, the form for $\Delta^{\prime}$ given by Eq. (19) reproduces reasonably accurately the numerical results for a cylindrical plasma obtained by Furth, Rutherford and Selberg. ${ }^{6}$

The instability properties of a particular rational surface are determined by the value of the current gradient parameter $\lambda$ [given in Eq. (15)] evaluated at the rational surface

$$
\lambda=-\frac{\mathrm{Iq} \mu_{\mathrm{o}} \bar{\sigma}}{2 \mathrm{mq^{ \prime }}} \frac{1}{\sqrt{-\overline{\mathrm{g}} \chi \chi_{\mathrm{g}}^{-} \psi \psi}} \equiv-\left.\frac{\mathrm{rq}}{\mathrm{m}(\mathrm{dq} / \mathrm{dr})} \frac{\mu_{\mathrm{o}}}{\mathrm{B}_{\theta}} \frac{\mathrm{dj}_{\|}}{\mathrm{dr}}\right|_{\mathrm{r}=\mathrm{r}_{\mathrm{s}}},
$$


where the first expression is written for toroidal equilibria and the second expression is valid for circular flux surface equilibria in a cylinder. The tearing mode matching parameter for zero pressure gradient plasmas is given by

$$
\Delta_{\mathrm{o}}^{\prime}=-2 \mathrm{~m} \sqrt{\overline{\mathrm{g}} \chi \chi} \lambda \pi \cot (\lambda \pi),
$$

where for circular flux surfaces $\sqrt{\bar{g} \chi \chi}=1 / r_{s}$. The asymptotically small order unity corrections to this leading order expansion are given in Appendix $C$. Tearing instability is indicated when $\Delta^{\prime}>0$, hence equation (46) predicts the condition $\lambda>0.5$ or

$$
1 \frac{\mathrm{Iq} \mu_{\mathrm{o}} \bar{\sigma}^{\prime}}{\mathrm{q}^{\prime}} \mid \frac{1}{\sqrt{\overline{\mathrm{g}}^{x x_{\mathrm{g}}^{-} \psi \psi}}}>\mathrm{m}
$$

as the threshold for a tearing instability with poloidal mode number $\mathrm{m}$. In the limit of circular flux surface plasmas this criterion is given by

$$
\left|\frac{q_{o} r_{s} \mu_{o}}{q_{o}^{\prime} B_{\theta}} \frac{d j_{\|}}{d r}\right|>m .
$$

As indicated in Figure 3, this threshold value is also predicted for plasmas with non-zero plasma gradient, however the value of $\Delta^{\prime}$ changes with $\beta$ away from the tearing threshold point. The saturated island width as obtained by the relation $\Delta^{\prime}\left(w_{\text {sat }}\right)=0$ can be found from the expression given in Eq. (23). As shown in Fig. 2 , this solution can be approximated to reasonable accuaracy by the relation

$$
\mathrm{w}_{\mathrm{sat}}=\frac{4.09}{2 \mathrm{~m}} \frac{1}{\sqrt{\frac{-}{\mathrm{g}} \chi \chi}}\left(\lambda-\frac{1}{2}\right) \cong \frac{2.04 \mathrm{r}_{\mathrm{s}}}{\mathrm{m}}\left(\lambda-\frac{1}{2}\right),
$$

where $w_{\text {sat }}$ is the saturated island half-width as a function of the parameter $\lambda$ and the second approximate solution is appropriate for circular flux surface plasmas. The appearance of a macroscopic magnetic island in a plasma equilibrium reduces the energy confinement time by a term proportional to the island width. $^{34}$ 
Since the asymptotic behavior of the ideal eigenfunctions is different in non-zero beta plasmas as opposed to zero pressure plasmas, the matching data for these two cases differ by a term proportional to the plasma pressure. In the guise of the Rutherford regime of tearing mode evolution, this difference of the matching data introduces a pressure gradient driven term in the island evolution equation. A simple scaling argument reproduces the results of Ref. 29, which derives the nonlinear analog of the Glasser effect of linear tearing mode theory. ${ }^{27,28}$ In this manner, the results of Sec. IV, reproduces the results of Ref. 30.

Although we have not tried to directly compare our tearing mode matching parameter to experimental profiles, a couple of observations can be made. The instability threshold, as predicted by Eq. (20), is easier to violate for lower mode numbers. Hence, it would seem unlikely that the appearance of a single island chain with helicity $\mathrm{m} / \mathrm{n}=4 / 3$ (or $5 / 4$ ), for instance, on TFTR can be explained by the $\Delta^{\prime}$ drive from tearing mode theory, since this theory says that the $n=2$ and $n=1$ modes should generally be more unstable. An effect not addressed in the present work is the bootstrap current free-energy source. ${ }^{31,32}$ The neoclassical pressure gradient term is larger than the pressure/curvature term in this work since it is of order $\beta_{\theta}=2 \mu_{o} p / B_{\theta}^{2}$ rather than the total $\beta=$ $2 \mu_{\mathrm{o}} \mathrm{p} / \mathrm{B}^{2}$ that entered here. ${ }^{33}$ Since fluctuations in the bootstrap current produce island producing instabilities in tokamaks, this effect could provide a possible explanation for the observed islands in high performance tokamak discharges. ${ }^{3}$

\section{Acknowledgments}

The authors would like to acknowledge useful conversations with Mike Hughes and Zuoyang Chang and thank Andrew Smolyakov for pointing out 
Refs. 22 and 24 to us. This work is supported by the U. S. DOE under grant no. DE-FG02-86ER53218.

\section{Appendix A: Whittaker Functions}

In this appendix, we compile some useful properties of Whittaker functions from Refs. 19 and 35. Whittaker functions are solutions of the differential equation

$$
\frac{\mathrm{d}^{2} \mathrm{u}}{\mathrm{d} \mathrm{x}^{2}}+\left(-\frac{1}{4}+\frac{\mathrm{k}}{\mathrm{x}}+\frac{1 / 4-\mu^{2}}{\mathrm{x}^{2}}\right) \mathrm{u}=0
$$

Equation (A1) has two linearly independent solutions given by

$$
\begin{aligned}
& \mathrm{M}_{\mathrm{k} / \mu}(\mathrm{X})=\mathrm{X}^{\mu+0.5} \mathrm{e}^{-\mathrm{X} / 2} \Phi(\mu-\mathrm{k}+1 / 2,2 \mu+1 ; \mathrm{X}) \\
& \mathrm{M}_{\mathrm{k} /-\mu}(\mathrm{X})=\mathrm{X}^{-\mu+0.5} \mathrm{e}^{-\mathrm{X} / 2} \Phi(-\mu-\mathrm{k}+1 / 2,-2 \mu+1 ; \mathrm{X})
\end{aligned}
$$

where

$$
\Phi(\alpha, \gamma ; X)=1+\frac{\alpha}{\gamma} \frac{X}{1 !}+\frac{\alpha(\alpha+1)}{\gamma(\gamma+1)} \frac{X^{2}}{2 !}+\frac{\alpha(\alpha+1)(\alpha+2)}{\gamma(\gamma+1)(\gamma+2)} \frac{X^{3}}{3 !}+\ldots
$$

is a degenerate hypergeometric function. When the parameter $2 \mu= \pm 1, \pm 2, \ldots$, the power series representation diverges, and these solutions are not valid. However, it is possible to introduce Whittaker's function, defined by

$$
\mathrm{W}_{\mathrm{k} / \mu}(\mathrm{X})=\frac{\Gamma(-2 \mu)}{\Gamma(1 / 2-\mu-\mathrm{k})} \mathrm{M}_{\mathrm{k} / \mu}(\mathrm{X})+\frac{\Gamma(2 \mu)}{\Gamma(1 / 2+\mu-\mathrm{k})} \mathrm{M}_{\mathrm{k} /-\mu}(\mathrm{X})
$$

which is also a solution of (A1) when $2 \mu$ approaches an integer. The functions $W_{k / \mu}(X)$ and $W_{-k / \mu}(-X)$ are linearly independent solutions. Whittaker's functions can be given by the integral relation

$$
W_{k / \mu}(X)=e^{-X / 2} \frac{X^{1 / 2-\mu}}{\Gamma(1 / 2-k+\mu)} \int_{0}^{\infty} d t e^{-t} t^{2 \mu-1}\left[1+\frac{X}{t}\right]^{k+\mu-1 / 2}
$$


A convenient relation derivable from (A6) is

$$
X \frac{\mathrm{d} W_{\mathrm{k} / \mu}}{\mathrm{dX}}=\left(\mathrm{k}-\frac{\mathrm{X}}{2}\right) \mathrm{W}_{\mathrm{k} / \mu}-\left[\mu^{2}-(\mathrm{k}-1 / 2)^{2}\right] \mathrm{W}_{\mathrm{k}-1 / \mu}
$$

For the special case of $\mu=1 / 2\left(\nu=\beta=0\right.$ in the tearing mode problem), $W_{k / \mu}$ has the small argument expansion

$$
\begin{aligned}
& W_{k / 0.5}(X)=\frac{\mathrm{e}^{-\mathrm{X} / 2}}{\Gamma(1-\mathrm{k})}\{1+ \\
&\left.\sum_{\mathrm{p}=0} \frac{\mathrm{kX}^{\mathrm{p}+1}}{\mathrm{p} !(1+\mathrm{p}) !} \frac{\Gamma(1-\mathrm{k}+\mathrm{p})}{\Gamma(1-\mathrm{k})}[\Psi(1+\mathrm{p})+\Psi(2+\mathrm{p})-\Psi(1-\mathrm{k}+\mathrm{p})-\ln \mathrm{X}]\right\}
\end{aligned}
$$

where $\Psi(x)=d \ln \Gamma(x) / d x$ is the Digamma-Psi function which satisfies the properties $\Psi(1+x)=\Psi(x)+1 / x$ and $\Psi(1-x)=\Psi(x)+\pi \cot (\pi x)$.

For $\mu=1 / 2+v$, with $v$ not an integer, the functions $M_{k / \mu}(X)$ and $M_{k /-\mu}(X)$ have a power series expansion at small $X$. Namely,

$$
\begin{aligned}
& M_{k / 0.5+v}(X)=X^{1+v}\left(1-\frac{k}{2(1+v)} X+\ldots\right), \\
& M_{k /-0.5-v}(X)=X^{-v}\left(1+\frac{k}{2 v} X+\ldots\right) ;
\end{aligned}
$$

$M_{k /-0.5-v}(X)$ represents the large solution as $X \rightarrow 0$, and $M_{k / 0.5+v}(X)$ represents the small solution.

\section{Appendix B: Stability of the Furth, Rutherford, and Selberg Currrent Profiles}

In the classic work by Furth, Rutherford and Selberg, ${ }^{6}$ the stability of a cylindrical, zero- $\beta$ plasma to tearing instability was examined. The set of current profiles of the form

$$
j_{||}(r)=\frac{j_{0}}{\left[1+(r / a)^{2 p}\right]^{1+1 / p}}
$$


was used, where $p=1,2,4$ describes peaked, rounded and flattened current profiles, respectively. Since the plasma is cylindrical, the poloidal magnetic field and $q$-profile are given by $B_{\theta}(r)=\left(j_{0} r / 2\right)\left[1+(r / a)^{2 p}\right]^{-1 / p}, q(r)=\left(2 B_{o} / R_{o b}\right)[1+$ $\left.(r / a)^{2 p}\right]^{1 / p}$, where $B_{o}$ is the strength of the axial magnetic field and $R_{o}$ is the periodicity length of the cylinder. Equation (21) predicts instability for the rational surface with poloidal mode number $m$ when the inequality given by

$$
\frac{2(1+p)}{\left[1+(r / a)^{2 p}\right]}>m
$$

is violated. The $\mathrm{q}$-profile is parameterized by the integer $\mathrm{p}$ and the value of $\mathrm{q}$ on the magnetic axis $q_{0}$. Consequently, Eq. (B2) can be rewritten as constraints on $\mathrm{q}_{\mathrm{o}}$ and $\mathrm{p}$. Instability occurs for the mode $\mathrm{m} / \mathrm{n}$ when the inequality

$$
2(1+p)\left(q_{0}\right)^{p}>\frac{m^{p+1}}{n^{p}}
$$

is satisfied. For the peaked profile $(p=1)$, modes with $m \geq 4$ are predicted to be always stable. The $\mathrm{m} / \mathrm{n}=2 / 1$ mode is unstable when $\mathrm{q}_{\mathrm{o}}>1$; the $\mathrm{m} / \mathrm{n}=3 / 2$ mode is unstable when $q_{0}>9 / 8$. The rounded profile $(p=2)$ has $m \geq 6$ modes always stable and the flat profile has $m \geq 10$ modes always stable. These observations are in basic agreement with the conclusions of Ref. 6 and the numerical results plotted in figures therein. Equation (B2) also indicates that the closer the rational surface is to the magnetic axis of the cylinder, the more likely instability will occur. Equation (B3) demonstrates that for this class of profiles, instability is more likely for larger values of $q_{0}$.

\section{Appendix C: Order $1 / \mathrm{m}$ corrections to $\Delta_{0}^{\prime}$}

For simplicity, we consider cylindrical plasmas, so that the minor radius serves as a flux function. Expanding the "inner" solution of Eq. (11) to first order in $1 / \mathrm{m}$ yields the equation 


$$
\frac{\mathrm{d}^{2} \mathrm{~A}_{1}}{\mathrm{dX} \mathrm{X}^{2}}-\left[\frac{1}{4}+\frac{\lambda}{\mathrm{X}}\right] \mathrm{A}_{1}=-\frac{1+\mathrm{c}}{2} \frac{\mathrm{dA} \mathrm{A}_{0}}{\mathrm{dX}}-X \frac{1+\mathrm{c}}{4} \mathrm{~A}_{0}-\lambda(\mathrm{b}+\mathrm{c}) \mathrm{A}_{0}
$$

where

$$
\begin{aligned}
& b=-\frac{r_{s}}{2} B_{\theta}\left(\frac{d j_{\mid 1}}{d r}\right)^{-1} \frac{d}{d r}\left(\frac{1}{B_{\theta}} \frac{d j_{\mid 1}}{d r}\right), \\
& c=r_{s}\left(\frac{d q}{d r}\right)^{-1} \frac{d^{2} q}{d r^{2}},
\end{aligned}
$$

are dimensionless quantities evaluated at the rational surface. Since $A_{0}$ satisfies Eq. (16), we can rewrite Eq. (C1) as

$$
\frac{1}{A_{0}} \frac{d}{d X} A_{0}^{2} \frac{d}{d X} \frac{A_{1}}{A_{0}}=-\frac{1+c}{2} \frac{d A_{0}}{d X}-X \frac{1+c}{4} A_{0}-\lambda(b+c) A_{0} .
$$

Integrating this equation twice, we obtain the solution

$$
\begin{aligned}
A_{1}(X)=A_{0}(X)\left\{C_{1}-X \frac{1+c}{4}\right. & \\
& \left.+\int_{0}^{X} d X^{\prime} \frac{1}{A_{0}^{2}\left(X^{\prime}\right)} \int_{ \pm \infty}^{X^{\prime}} d X^{\prime \prime}\left[-X^{\prime \prime} \frac{1+c}{4}-\lambda(b+c)\right] A_{0}^{2}\left(X^{\prime \prime}\right)\right\}
\end{aligned}
$$

where the boundary condition $A_{1}(0)=C_{1} A_{0}(0)$ is used and the $+\infty(-\infty)$ boundary of the integral refers to the solutions when $X>0(<0)$. The matching parameter to first order in $1 / \mathrm{m}$ is given by

$$
\Delta^{\prime}=\Delta_{o}^{\prime}\left(1-\frac{C_{1}}{m}\right)+\frac{1}{m A_{0}(0)} \lim _{\varepsilon \rightarrow 0}\left\{\frac{\mathrm{dA}_{1}}{d r}\left(r_{s}+\varepsilon\right)-\frac{d_{1}}{d r}\left(r_{s}-\varepsilon\right)\right\},
$$

where $\Delta_{\mathrm{o}}^{\prime}$ is the matching parameter of the lowest order solution, Eq. (19). From Eq. (C5), it is easy to show that $\Delta^{\prime}$ to first order in $1 / \mathrm{m}$ is given by

$$
\Delta^{\prime}=\Delta_{0}^{\prime}+\frac{2}{r} f_{R}(0+)-f_{L}(0-)
$$

where 


$$
\begin{aligned}
& f_{R}(0+)=\int_{\infty}^{0} d X\left[-X \frac{1+c}{4}-\lambda(b+c)\right] A_{0 R}^{2}(X), \\
& f_{L}(0-)=\int_{-\infty}^{0} d X\left[-X \frac{1+c}{4}-\lambda(b+c)\right] A_{0 L}^{2}(X),
\end{aligned}
$$

in which $A_{O R}(X)$ and $A_{0 L}(X)$ refer to the leading order solutions for $X>0$ and $X<$ 0 , respectively. The first term, $\Delta_{0}^{-}$, is defined in Eq. (18) and given by the expression $\Delta_{0}^{\prime}=-(2 \mathrm{~m} / \mathrm{r}) \pi \lambda \cot (\pi \lambda)$ for cylindrical plasmas. Substituting Eq. (17), we obtain the matching condition to first order in $1 / \mathrm{m}$,

$$
\begin{aligned}
\frac{1 \Delta^{\prime}}{2 \mathrm{~m}}=- & \pi \lambda \cot (\pi \lambda)-\frac{1+\mathrm{c}}{\mathrm{m}} \int_{0}^{\infty} \mathrm{d} X \frac{\mathrm{X}}{4}\left\{\Gamma^{2}(1-\lambda) \mathrm{W}_{\lambda / 0.5}^{2}(\mathrm{X})-\Gamma^{2}(1+\lambda) \mathrm{W}_{-\lambda / 0.5}^{2}(\mathrm{X})\right\} \\
+ & \frac{\lambda(\mathrm{b}+\mathrm{c})}{\mathrm{m}} \int_{0}^{\infty} \mathrm{dX}\left\langle\Gamma^{2}(1+\lambda) \mathrm{W}_{-\lambda / 0.5}^{2}(\mathrm{X})+\Gamma^{2}(1-\lambda) \mathrm{W}_{\lambda / 0.5}^{2}(\mathrm{X})\right\} . \quad(\mathrm{C} 10)
\end{aligned}
$$

The integrals can be reduced to functions of the parameter $\lambda$. After a lengthy calculation, Eq. (C10) is given by the simplified form

$$
\begin{aligned}
\frac{r \Delta^{\prime}}{2 m}=-\pi \lambda \cot (\pi \lambda) & +\frac{b+c}{m} \frac{2 \pi^{2} \lambda^{3}}{\sin ^{2}(\pi \lambda)} \\
& -\frac{1+c}{m} \frac{3 \lambda}{2}\left\{1+\lambda^{2}\left[\Psi^{\prime}(1-\lambda)-\Psi^{\prime}(1+\lambda)\right]\right\}
\end{aligned}
$$

where $\Psi^{\prime}(x)=d \Psi(x) / d x$ is the derivative of the Digamma-Psi function. The last term is negative if $\lambda(1+c)>0$ and is therefore stabilizing for $c>-1$. The second term is destabilizing (stabilizing) if $\lambda(b+c)$ is positive (negative). Near the leading order stability threshold $\lambda=1 / 2$, the $1 / \mathrm{m}$ corrections can be evaluated. The matching condition when $\lambda$ is close to 0.5 is given by

$$
\frac{\mathrm{r} \Delta^{\prime}}{2 \mathrm{~m}} \cong \frac{\pi^{2}}{2}\left(\lambda-\frac{1}{2}\right)+\frac{1}{\mathrm{~m}}\left[\frac{\pi^{2}}{4}(\mathrm{~b}+\mathrm{c})-\frac{3}{2}(1+\mathrm{c})\right] .
$$


Consequently, the correction terms change the marginal stability condition slightly. It should be noted that for $\lambda$ close to unity, the correction factors get quite large. In fact, depending on the values for $b$ and $c$, these terms may become comparable to the leading order $\Delta^{\prime}$, which suggests that the perturbation theory presented here may not be accurate.

For the profiles of Appendix $B, b+c=2+(p-2)\left(q_{0} / q\right)^{p}$ and $c+1=2+$ $(2 p-2)\left(q_{o} / q\right)^{p}$ for the mode resonant at the $m / n=q$ surface and $q_{o}$ is the value of $\mathrm{q}$ on axis. For these profiles, thus the last term is stabilizing and the second term is destabilizing. 


\section{References}

1. H. P. Furth, J. Killeen, and M. N. Rosenbluth, Phys. Fluids 6, 459 (1963).

2. J. D. Callen, B. V. Waddell, B. Carreras, M. Azumi, P. J. Catto, H. R. Hicks, J. A. Holmes, D. K. Lee, S. J. Lynch, J. Smith, M. Soler, K. T. Tsang, and J. C. Whitson, in Plasma Physics and Controlled Nuclear Fusion Research 1978, Innsbruck (IAEA, Vienna, 1979), Vol. 1, p. 415.

3. Z. Chang, E. D. Fredrickson, J. D. Callen, K. M. McGuire, M. G. Bell, R. V. Budny, C. E. Bush, D. S. Darrow, A. C. Janos, L. C. Johnson, H. Park, S. D. Scott, J. D. Strachan, E. J. Synakowski, G. Taylor, R. M. Wieland, M. C. Zarnstorff, S. J. Zweben and the TFTR Team, to be submitted to Nucl. Fuison (1993); Princeton Plasma Physics Laboratory Report No. PPPL-2941 (October, 1993).

4. E. J. Strait, Bull. Am. Phys. Soc. 38, 2098 (1993).

5. Z. Chang and J. D. Callen, Nucl. Fusion 30, 219 (1990).

6. H. P. Furth, P. H. Rutherford, and H. Selberg, Phys. Fluids 16, 1054 (1973).

7. A. E. Adler, R. M. Kulsrud, and R. B. White, Phys. Fluids 23, 1375.

8. A. Bondeson and J. R. Sobel, Phys. Fluids 27, 2028 (1984).

9. J. F. Drake and Y. C. Lee, Phys. Fluids 20, 1341 (1977).

10. P. H. Rutherford, Phys. Fluids 16, 1903 (1973).

11. J. F. Drake and Y. C. Lee, Phys. Rev. Lett. 39, 453 (1977).

12. R. B. White, D. A. Monticello, M. N. Rosenbluth, and B. V. Waddell, Phys. Fluids 20, 800 (1977).

13. B. Carreras, B. V. Waddell, and H. R. Hicks, Nucl. Fusion 19, 1423 (1979).

14. B. Coppi, J. M. Greene, J. L. Johnson, Nucl. Fusion 6, 101 (1966).

15. A. Pletzer and R. L. Dewar, J. Plasma Phys. 45, 427 (1991).

16. M. S. Chu, R. L. Dewar, J. M. Greene, and A. Pletzer, Phys. Fluids B 5, 1593 (1993). 
17. J. W. Connor, S. C. Cowley, R. J. Hastie, T. C. Hender, A. Hood, and T. J. Martin, Phys. Fluids 31, 577 (1988).

18. J. P. Friedberg, Ideal Magnetohydrodynamics, Plenum Press, New York, pp. 136-147, (1987).

19. W. Magnus, F. Oberhettinger, and R. P. Soni, Formulas and Theorems for the Special Functions of Mathematical Physics, (Springer-Verlag, New York, 1966), pp. 295-323.

20. H. R. Strauss, Phys. Fluids 24, 2004 (1981).

21. J. A. Wesson, Nucl. Fusion 18, 87 (1978).

22. N. V. Ivanov, Sov. J. Plasma Phys. 9, 407 (1983),

23. P. H. Rutherford, in "From Particles to Plasmas," J. W. Van Dam ed. (Addison-Wesley, New York, 1987), pp. 249-271.

24. B. N. Kuvshinov and A. B. Mikhailovskii, Sov. J. Plasma Phys. 16, 639 (1991).

25. A. Thyagaraja, Phys. Fluids 24, 1716 (1981).

26. R. Pellat, M. Frey and M. Tagger, J. Physique 45, 1615 (1984).

27. A. H. Glasser, J. M. Greene, and J. L. Johnson, Phys. Fluids 18, 875 (1975); Phys. Fluids 19, 567 (1976).

28. T. C. Hender, R. J. Hastie, and D. C. Robinson, Nucl. Fusion 27, 1389 (1987).

29. M. Kotschenreuther, R. D. Hazeltine, and P. J. Morrison, Phys. Fluids 28, 294 (1985).

30. C. M. Bishop, J. W. Connor, R. J. Hastie, and S. C. Cowley, Plasma Phys. Controlled Nucl. Fusion 33, 389 (1991).

31. R. Carrera, R. D. Hazeltine, and M. Kotschenreuther, Phys. Fluids 29, 899 (1986); see also National Technical Information Service Document No. DE86008946 (UW Report UWPR 85-5 by W. X. Qu and J. D. Callen). 
Copies may be ordered from the National Technical Information Service, Virginia 22161. The price is $\$ 12.50$ plus a $\$ 3.00$ handling fee. All orders must be prepaid.

32. C. C. Hegna and J. D. Callen, Phys. Fluids B 4, 1855 (1992).

33. C. C. Hegna and A. Bhattacharjee, Phys. Fluids B 2, 1804 (1990).

34. Z. Chang and J. D. Callen, Nucl. Fusion 30, 219 (1990).

35. I. S. Gradshteyn and I. M. Ryzhik, Tables of Integrals, Series, and Products, (Academic Press, Orlando, 1980), pp. 1059-1063. 


\section{Figure Captions}

Fig. 1 - Eigenfunctions as given by Eq. (17) as a function of the strained variable $X$. The three plots are given for the values $\lambda=0.4, \lambda=0.6$, and $\lambda=0.8$. As the parameter $\lambda$ becomes larger the difference in the derivative across $X=0$ becomes larger, indicating a larger $\Delta^{\prime}$.

Fig. 2 - The solution of the condition $\Delta^{\prime}\left(\omega_{\text {sat }}\right)=0$ as a function of $\lambda$ from the solution to Eq. (23) for $\lambda>0.5$ (solid line). Also plotted is the analytic fit (dashed line), $\omega=4.09(\lambda-0.5)$, which reproduces the more complex expression quite well.

Fig. 3 - The functions $\Delta_{\beta}^{\prime}$ versus $\lambda$ for a number of different values of $v$. They are denoted by $v=0$ (solid line), $v=0.1$ (long dashes), $v=0.2$ (short dashes), $v=0.3$ (long dash-dots), $v=0.4$ (short dash-dots). Note that the threshold condition for all these cases is the same $\lambda=0.5$ as it is in the zero beta case. 

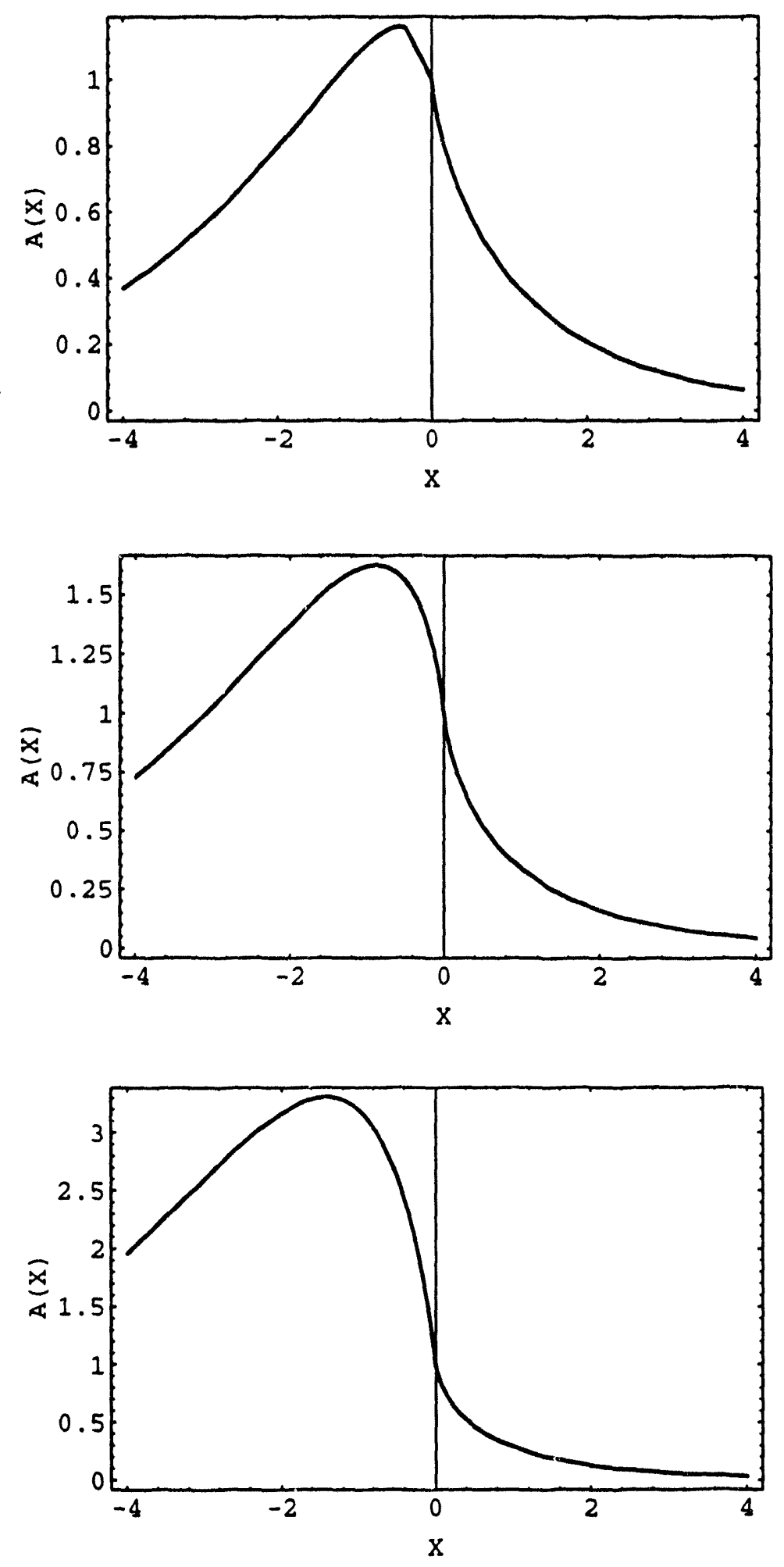

Fig. 1 


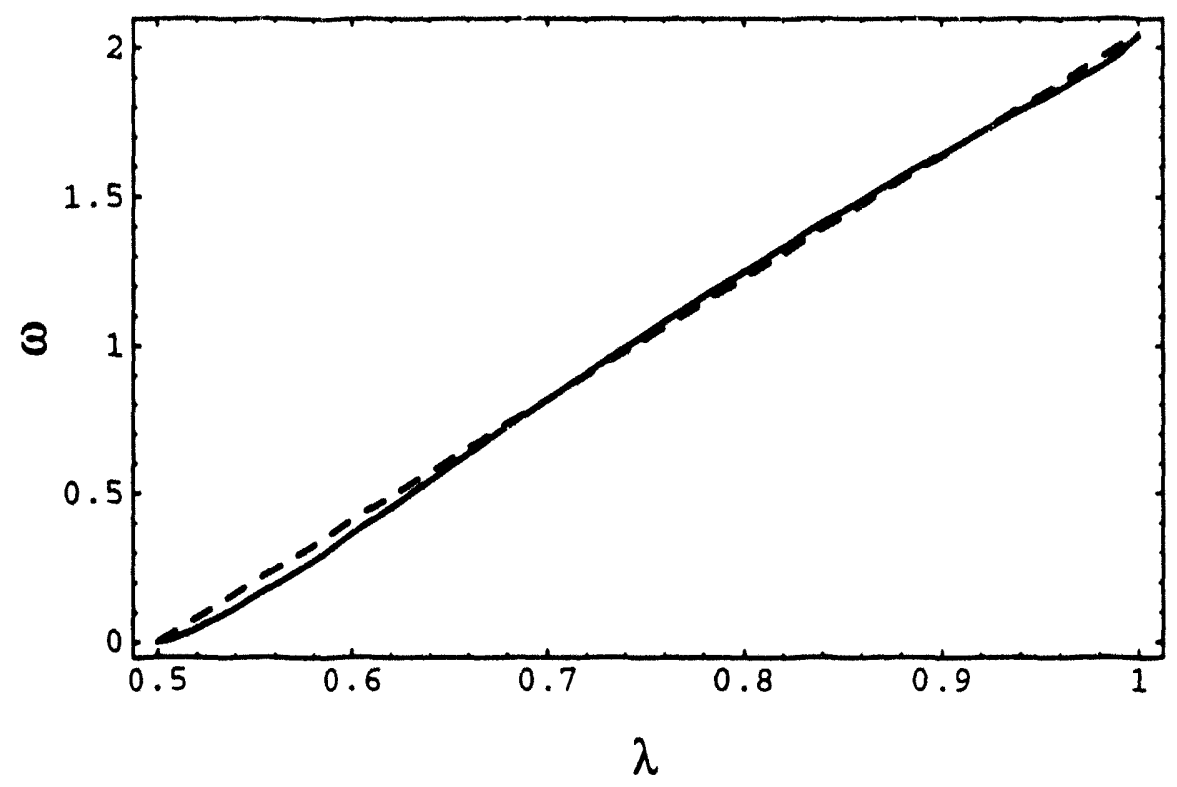

Fig. 2 


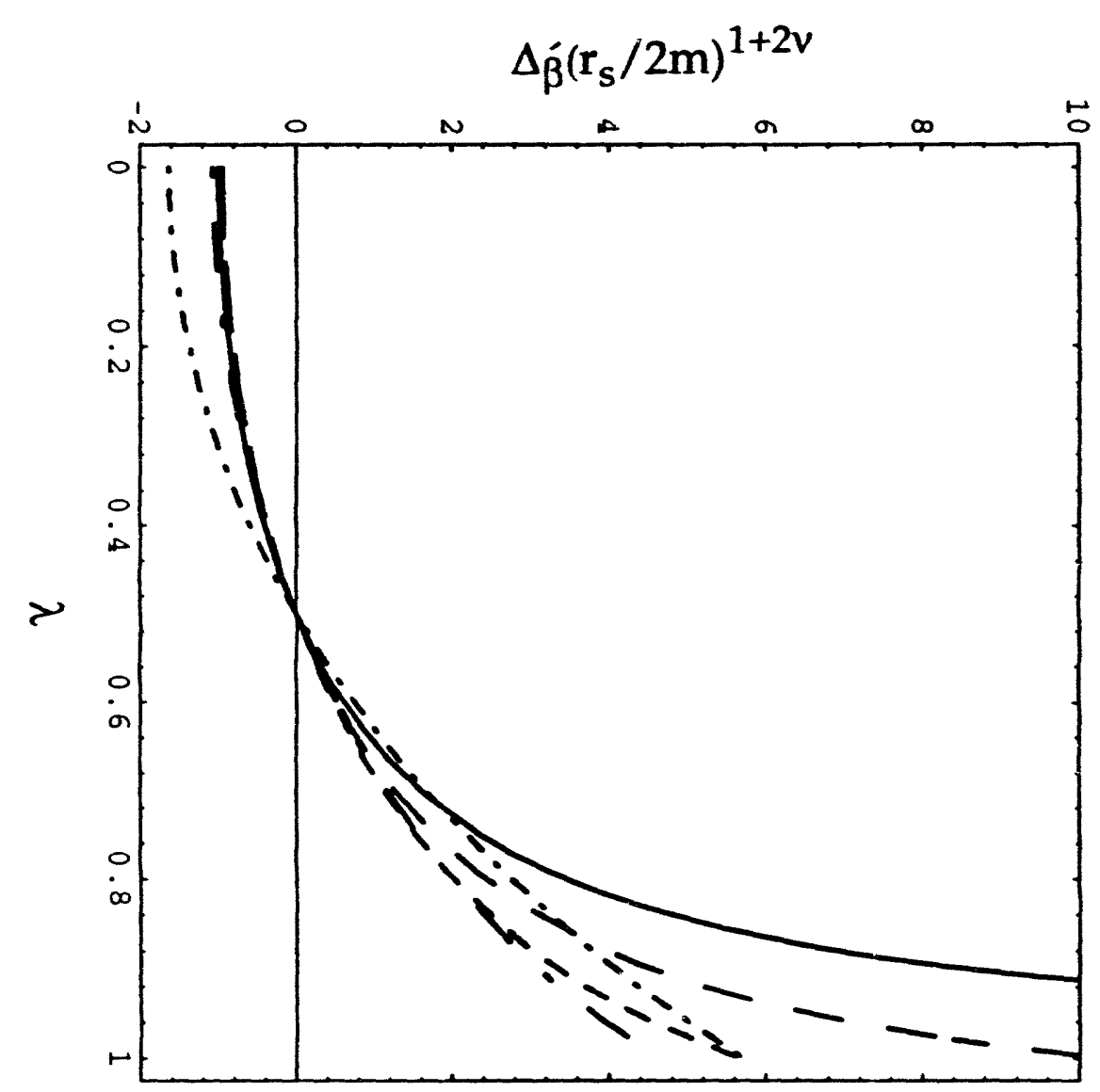



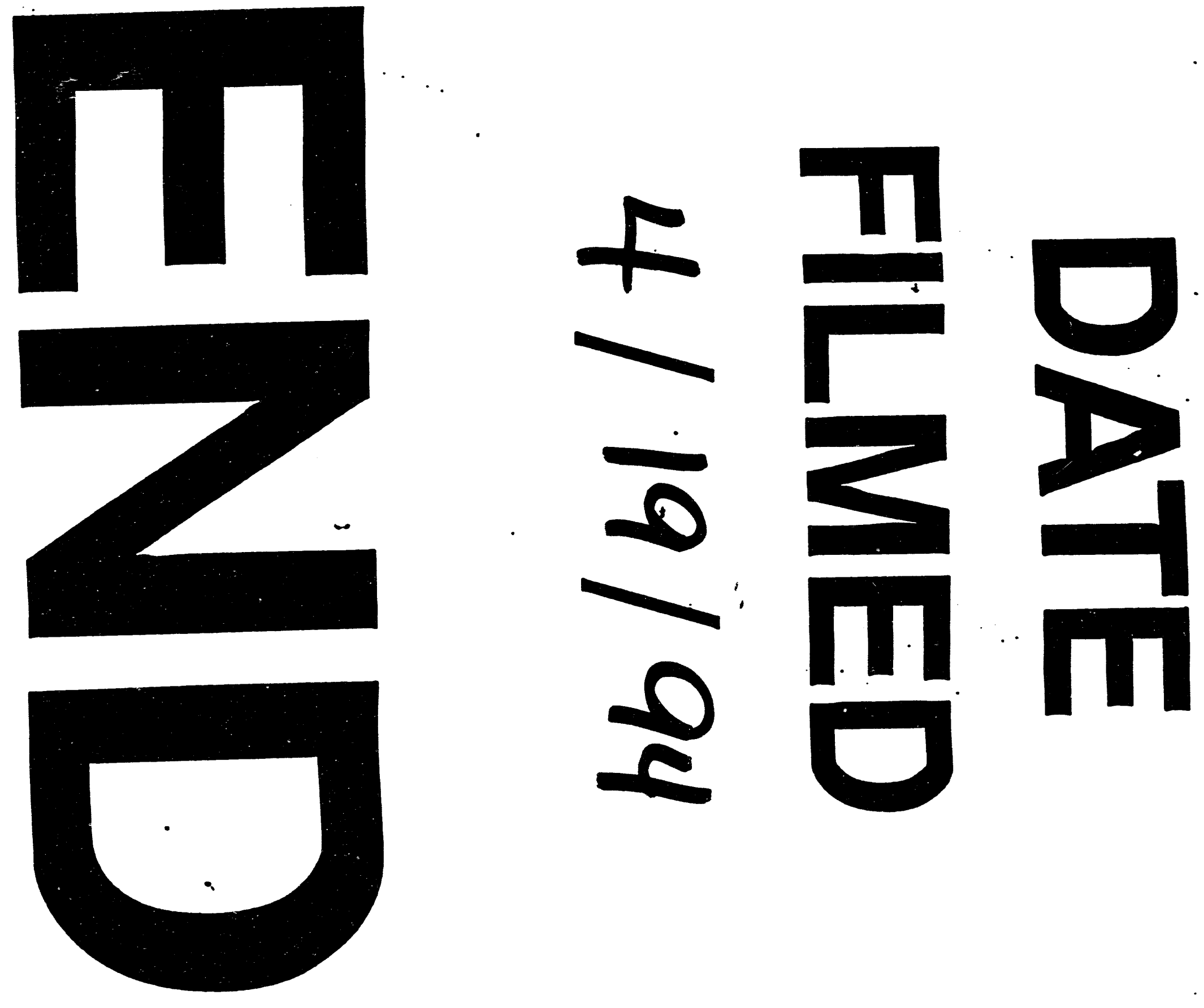
